\title{
Routing optimization of the emergency supplies distribution vehicles using NSGA-II algorithm: a case study
}

\author{
Xingjiang $\mathrm{Li}^{1,2}$, Hanyun $\mathrm{Yin}^{3}$ and Fuhai $\mathrm{Yan}^{2, \mathrm{a}}$ \\ ${ }^{1}$ The Economy and Enterprise Development Institute, Sichuan University ,Chengdu 610065, China \\ ${ }^{2}$ Business School, Sichuan University, Chengdu 610065, China \\ ${ }^{3}$ Chengdu University of Technology, Chengdu 610059, China
}

\begin{abstract}
In recent years, emergencies, including natural disasters and other public disasters, have seriously threatened the lives and property security of people all over the world. In order to save more people's lives and reduce the losses caused by disasters, many researchers have carried out intensive study on the distribution of emergency supplies. This paper first studies Location-Routing Problem(LRP) of alternative logistics centers and material demand points, and constructs a multi-objective integer programming model based on the actual situation. The model consists of two objectives: (1) the minimum total transportation time; (2) the maximum total emergency material satisfaction. Then an algorithm is introduced to solve the above model: NSGA-II. Finally, the emergency materials distribution in Hubei Province is taken as an example to verify the applicability and effectiveness of the above method and the models.
\end{abstract}

\section{Introduction}

In recent years, earthquakes, floods, hurricanes, tsunamis and other emergencies continue to cause massive loss of life and property [1], causing widespread concern around the world. And the distribution of emergency materials after disasters has also been highly valued by the academic community [2]. Due to the huge casualties and property losses caused by emergencies, a large number of emergency materials are in urgent need to deal with the problems such as the rescue of the wounded, the reconstruction after the disaster, the prevention of epidemic, the recovery of production, and the restoration of order. The effective distribution of post-disaster emergency rescue plays an important role in the postdisaster rescue operation, and the location of alternative logistics centers and the route selection of vehicles in the existing transportation network are the two of the most challenging issues in the emergency logistics [3]. In the post-disaster emergency material distribution system, issues like the location of alternative logistics centers, the ownership of transportation vehicles, the optimization of emergency material distribution route, and the distribution of emergency materials need to be solved systematically [4].

Scholars in different disciplines have designed many models and algorithms to solve the problem of emergency material distribution. Zhou Yawen designed a multi-objective optimization model, put forward a multiobjective evolution algorithm framework based on decomposition, and used many examples and experiments to verify the applicability of the algorithm in large-scale disasters [5]. Xu Jiuping put forward a double-layer model under random fuzzy environment for emergency material scheduling within 72 hours after the earthquake, and designed an interactive genetic algorithm to search the optimal solution of the double-layer model [6]. The above two scholars mainly study the distribution of emergency rescue materials after the earthquake. Behnam Vahdan proposed a new multi-objective model and proposed two meta heuristic algorithms: nondominated sorted genetic algorithms-II (NSGA-II) and multi-objective particle swarm optimization algorithm (MOPSO). The model reduces the delivery time and total cost to the maximum extent, and improves the reliability of the route [7]. Li Qiuping proposed a multi-objective rescue path model of urban emergency logistics based on travel time reliability, designed a hybrid meta heuristic algorithm combining ant colony optimization (ACO) and tabu search (TS) to solve the model, and proved that the method can improve the rescue efficiency and high travel time reliability through the rescue path optimization experiment under the actual urban storm event [8]. By studying the methods and processes of Behnam vahdan and Li Qiuping, we find that time is a very important factor in the distribution of emergency materials and we should try our best to save the delivery time and ensure the reliability of the delivery time.

Through the above literature review, it can be found that scholars at home and abroad have conducted extensive research on LRP under different disaster backgrounds, and most scholars focus on natural disasters

\footnotetext{
$\overline{{ }^{a} \text { Corresponding author: fuhai_yan @ } 126 . c o m}$
} 
such as earthquake [9] and flood [10]. However, there is few research on the distribution of emergency materials for public health emergencies. This paper will use NSGA-II algorithm to solve the emergency material distribution problem of public health emergencies caused by infectious diseases.

\section{Model formulations}

\subsection{Problem description}

In order to reduce the major loss of public health caused or might be caused by public health emergencies, the government may take isolation and restriction measures in the areas with serious epidemic situation. At the same time, in order to prevent further spread of the epidemic and meet the basic living security needs of the people in the isolation area, distribution of emergency materials is of necessity. In the decision-making process of this paper, the objectives to be optimized are: (1) transportation time: due to the urgency of the emergency demand in the epidemic isolation area, the distribution time of emergency materials is required to be as short as possible; (2) material satisfaction: under the condition of limited emergency materials and means of transportation, the material demand in the epidemic isolation area should be satisfied as much as possible.

\subsection{Assumptions}

Here are some assumptions used in our model:

(1) The location and quantity of the material demand points in the epidemic isolation area are known;

(2) The location and number of alternative logistics centers are known, and there is no capacity limit;

(3) Use the same type and number of vehicles with known capacity limits;

(4) The vehicle will not return the emergency materials after they are delivered to the demand points of the final service;

(5) The construction quality of the highway, including the design speed, lane width, route crossing and service level, meets the relevant technical requirements; and these indicators are known.

(6) Only ground road transportation time is considered, and the time used for loading and unloading materials at each node is not considered.

\subsection{Notations and definitions}

The symbols used in the following text are listed as follows:

(1) Set and indices

$M$ : Number set of alternative logistics centers, $M=\{1,2, \ldots, m\}$;

$N$ : Number set of material demand points, $N=\{m+1$, $m+2, \ldots m+n\}$;

$P$ : Number set of nodes, $P=M \cup N$;

$i, j$ : Path node, $i, j \in P$;

$E$ : Path link set, $E=\{(i, j), i, j \in P, i \neq j\}$;
$K$ : Number set of Transport vehicle, $K=\{1,2, \ldots, k\}$;

$k$ : Transport vehicle No. $k, k \in K$.

(2) Parameters

$m$ : Number of alternative logistics centers;

$n$ : Quantity of material demand points;

$k$ : Number of transport vehicles;

$t_{i j}$ : Transportation time of vehicle through link $(i, j)$, $(i, j) \in E$;

$Q_{i}$ : Quantity of emergency materials required at point $i, i \in N$;

$Q:$ Total amount of emergency materials in logistics system;

$U W$ : Weight of each emergency material;

$W$ : Load per vehicle.

(3) Decision variables

$x_{i}: 1$, If the alternative logistics center is set at $i, 0$ otherwise;

$y_{i j k}: 1$, If $i$ is the previous point of $\mathrm{j}$ on the path of vehicle $k, 0$ otherwise, $(i, j) \in E, k \in K$;

$Z_{i k}: 1$, If node $i$ is on the path of vehicle $k, 0$ othsewise, $i \in P, k \in K$;

$q_{i k}$ : Quantity of emergency materials transported by vehicle $k$ to demand point $i, i \in N, k \in K$;

$U_{i k}: 1$, If the last arriving node of vehicle $k$ is $i, 0$ otherwise, $i \in P, k \in K$.

\subsection{Multi-objective mathematical model}

Since the time taken by vehicles to load and unload materials at each node is not considered, the objective function 1 is to minimize the total transportation time of all routes:

$$
f_{1}=\min \sum_{k \in K} \sum_{(i, j) \in E} t_{i j} y_{i j k}
$$

The material satisfaction degree of the material demand point $\mathrm{i}$ is $\frac{\sum_{k \in K} q_{i k} Z_{i k}}{Q_{i}}$ and the objective function 2 is to maximize the sum of material satisfaction at the demand points:

$$
f_{2}=\max \sum_{i \in N} \frac{\sum_{k \in K} q_{i k} z_{i k}}{Q_{i}}
$$

The constraints are as follows :

$$
\begin{aligned}
& x_{i} \geq y_{i j k}, i \in M,(i, j) \in E, k \in K \\
& x_{i} \geq z_{i k}, i \in M, k \in K \\
& z_{i k}+z_{j k} \leq 1, i, j \in M \\
& \sum_{i \in N} u_{i k}=1, k \in K \\
& \sum_{i \in M} \sum_{k \in K} \sum_{j \in N} q_{i k} z_{i k} \leq Q \\
& \sum_{k \in K} q_{i k} z_{i k} \leq Q_{i}, i \in N \\
& \sum_{k \in K} \sum_{j \in P} y_{i j k} \geq 1, i \in N \\
& \sum_{i \in M} \sum_{j \in N} y_{i j k} \leq 1,(i, j) \in E, k \in K \\
& \sum_{i \in P} y_{i j k} \leq 1, i \in N, k \in K \\
& q_{i k} \geq 0, k \in K, i \in N \\
& \sum_{i \in N} U W q_{i k} \leq W, k \in K \\
& x_{i} \in(0,1), i \in M \\
& y_{i j k} \in(0,1),(i, j) \in E, k \in K \\
& z_{i k} \in(0,1), i \in P, k \in K \\
& u_{i k} \in(0,1), i \in N, k \in K
\end{aligned}
$$

Constraint (3) indicates that if and only if node $i$ is on the route, the route will go directly from node $i$ to the node j; Constraint (4) indicates that only the selected 
logistics distribution center can provide materials; Constraint (5) indicates that any two logistics distribution centers cannot be connected; Constraint (6) indicates that each vehicle can only stay at one node at the end; Constraint (7) indicates that the quantity of materials transported to the demand points cannot exceed the total quantity of existing materials; Constraint (8) indicates the upper limit of materials distributed to each demand point; Constraint (9) ensure that each demand point is visited at least once; Constraint (10) ensure that each vehicle can only start from one logistics distribution center; Constraint (11) indicate that each demand point can only be visited by the same vehicle at most once; Constraint (12) indicate that the materials delivered to demand points i cannot be negative; Constraint (13) indicate that the total weight of materials delivered by each vehicle cannot exceed the load of the vehicle; Constraints (14)(17) indicate that the value range of variable can only be 0 or 1 .

\section{Introduction of algorithm}

\subsection{NSGA-II}

In this paper, NSGA-II is proposed to solve the model. NSGA-II is suitable for complex and multi-objective optimization problems [11]. Compared with NSGA, NSGA-II improves the following three aspects: (1) the computational complexity of NSGA is $O\left(M N^{3}\right)$, and the sorting speed is very slow when the population size is large. NSGA-II proposed the concept of fast non dominated sorting, which reduced the computational complexity to $O\left(M N^{2}\right)$ and greatly improved the performance of the algorithm. (2) The elitist strategy is used to ensure that the optimal solution is not lost and improve the search performance. (3) NSGA-II adopts crowding degree measurement operator to overcome the defect of artificially specifying shared parameters in NSGA.

\subsection{Algorithm design}

According to the structural characteristics of the solution, this paper adopts the natural number coding method, as shown in formula (18). And each chromosome contains four substrings.

$$
P_{g}^{t}=\left\{P_{g 1}^{t}, P_{g 2}^{t}, P_{g 3}^{t}, P_{g 4}^{t}\right\}
$$

The length of the first substring $P_{g 1}^{t}$ is $\alpha$, which represents the number of vehicles in the model. And the number of each gene bit is a random natural number from 1 to $m$, and $m$ is the number of alternative logistics centers. The substring determines the location of the alternative logistics centers and the ownership of the vehicles.

The length of the second substring $P_{g 2}^{t}$ is also $\alpha$, and the number of each gene bit is a random natural number from 1 to $\alpha$, which indicates the vehicle number. The length of the third substring $P_{g 3}^{t}$ is $\beta$, which is randomly arranged by natural numbers from 1 to $\alpha$. The second substring ensures that every vehicle will be on duty to deliver goods, and the third substring ensures that every demand point has vehicles to deliver goods. The length of the fourth substring $P_{g 4}^{t}$ is $\gamma(\gamma=\alpha+\beta)$. And $\gamma$ represents the quantity of material demand points, and its sequence from front to back is the service sequence of corresponding vehicles. The four sub strings jointly determine the transportation route of the vehicle.

For example, the number of alternative logistics centers is $\{1,2\}$, the vehicle number is $\{1,2,3,4,5,6,7,8,9$, $10\}$, the number of material demand points is $\{3,4,5,6,7,8,9,10,11,12,13,14,15,16\}$, on the chromosome $\{1,2,1,2,1,1,2,2,1,1,3,5,2,7,1,4,6,8,9,10,8,5,3,7,13,5,4,8,9$, $11,12,15,16,3,6,7,10,14\}$, the first substring is $\{1,2,1,2,1,1,2,2,1,1\}$, and the location and vehicle ownership of its logistics center are shown in Table 1:

Table 1. Location of alternative logistics centers and ownership of vehicles

\begin{tabular}{|c|c|c|c|c|c|c|c|c|c|c|}
\hline $\mathbf{K}$ & $\mathbf{1}$ & $\mathbf{2}$ & $\mathbf{3}$ & $\mathbf{4}$ & $\mathbf{5}$ & $\mathbf{6}$ & $\mathbf{7}$ & $\mathbf{8}$ & $\mathbf{9}$ & $\mathbf{1 0}$ \\
\hline $\mathbf{M}$ & 1 & 2 & 1 & 2 & 1 & 1 & 2 & 2 & 1 & 1 \\
\hline
\end{tabular}

The second substring is $\{3,5,2,7,1,4,6,8,9,10\}$, the third is $\{8,5,3,7\}$, the fourth is $\{13,5,4,8,9,11,12,15,16,3$, $6,7,10,14\}$, which jointly determine the demand points of vehicle service. The specific correspondence is shown in Table 2 and table 3:

Table 2. Correspondence between vehicles and material demand points

\begin{tabular}{|c|c|c|c|c|c|c|c|c|c|c|}
\hline $\mathbf{K}$ & $\mathbf{3}$ & $\mathbf{5}$ & $\mathbf{2}$ & $\mathbf{7}$ & $\mathbf{1}$ & $\mathbf{4}$ & $\mathbf{6}$ & $\mathbf{8}$ & $\mathbf{9}$ & $\mathbf{1 0}$ \\
\hline $\mathbf{N}$ & 13 & 5 & 4 & 8 & 9 & 11 & 12 & 15 & 16 & 3 \\
\hline
\end{tabular}

Table 3. Correspondence between vehicles and material demand points

\begin{tabular}{|c|c|c|c|c|}
\hline $\mathbf{K}$ & $\mathbf{8}$ & $\mathbf{5}$ & $\mathbf{3}$ & $\mathbf{7}$ \\
\hline $\mathbf{N}$ & 6 & 7 & 10 & 14 \\
\hline
\end{tabular}

The transportation route of vehicles is jointly determined by four substrings, that is, the vehicles represented by genes in substring 2 and substring 3 start from the logistics distribution center determined by substring 1 , and then send the correspondding material demand points in substring 4 in turn. The vehicle route determined by this chromosome is shown in Table 4:

Table 4. Vehicle transportation route

\begin{tabular}{|c|c|c|}
\hline $\mathbf{K}$ & $\mathbf{M}$ & Transportation route \\
\hline $\mathbf{1}$ & 1 & $1-9$ \\
\hline $\mathbf{2}$ & 2 & $2-4$ \\
\hline $\mathbf{3}$ & 1 & $1-13-10$ \\
\hline $\mathbf{4}$ & 2 & $2-11$ \\
\hline $\mathbf{5}$ & 1 & $1-5-7$ \\
\hline $\mathbf{6}$ & 1 & $1-12$ \\
\hline $\mathbf{7}$ & 2 & $2-8-14$ \\
\hline $\mathbf{8}$ & 2 & $2-15-6$ \\
\hline $\mathbf{9}$ & 1 & $1-16$ \\
\hline $\mathbf{1 0}$ & 1 & $1-3$ \\
\hline
\end{tabular}

\subsection{Stages of the algorithm}

The main flow of the algorithm designed in this paper is 
as follows (this paper does not explain the process of selection, crossover or variation):

Step 1: the initial population $P_{0}$ with the scale of $N$ is generated, and its maximum evolution algebra is $T$;

Step 2: carry on the genetic operation to the population $P t$, produce the new chromosome population Qt with the scale of $N$, and merge it into the new population $R t$ with the scale of $2 N$;

Step 3: calculate the non dominated ranking of $R t$, and get the non dominated level and crowding degree of each individual;

Step 4: according to the crowding operator, the chromosome individuals with size $N$ are selected to enter the next generation population $P t+1$;

Step 5: if $t$ is less than the maximum number of iterations $T$, return to step 2; otherwise, the algorithm ends.

\section{Case study}

COVID-19 broke out worldwide in 2020 and China is seriously affected by the epidemic. Among all the provinces in China, Hubei is the most severely affected. For the sake of in epidemic prevention and control, the governments of all regions in Hubei Province took measures to "close down cities" within a week of the outbreak. This paper assumes that in order to strengthen the prevention and control of the epidemic in Hubei Province, the Chinese government plans to urgently dispatch a batch of protective materials (disposable ordinary masks) from the whole country to support the fight against the epidemic in Hubei Province. According to the material support information published on the portal of Hubei Provincial People's government, Xiangyang and Ezhou City are selected as the two alternative logistics centers, and the rest areas are all material demand points (Since Wuhan is seriously affected by the epidemic, in order to reduce the probability of cross infection, the materials needed for Wuhan should be solved separately, and it is not considered to be an alternative logistics center or a material demand point).

\subsection{Information of Materials and vehicles}

Table 5 shows the information of the protective materials to be distributed and the specification and quantity of distribution vehicles to be used this time.

Table 5. Information of emergency materials and vehicles

\begin{tabular}{|c|c|c|c|}
\hline Emergency & Material name & UW & Quantity \\
\cline { 2 - 4 } matetials & Mask & $10 \mathrm{~kg}$ & 7500 boxes \\
\hline \multirow{2}{*}{ Vehicles } & Weight Load & $\begin{array}{c}\text { Number of } \\
\text { vehicles }\end{array}$ & $\begin{array}{c}\text { Travel } \\
\text { speed }\end{array}$ \\
\cline { 2 - 4 } & $\mathbf{1 0 M T}$ & 10 & $80 \mathrm{~km} / \mathrm{h}$ \\
\hline
\end{tabular}

\subsection{Information of material demand points}

Table 6 shows the distance between each point (Relevant distance information is obtained from Baidu map, unit: $\mathrm{km}$. The cities represented by the figures are as follows: 1-Xiangyang, 2-Ezhou City, the cities represented by number 3-16 are shown in Table 7)

Since the protective materials transported this time are necessary for epidemic prevention, in order to meet the epidemic prevention needs of all regions in Hubei Province, this paper plans to distribute materials according to the proportion of population distribution in each region. With reference to the proportion of population in each region of Hubei Province (data source of population proportion: Statistical Yearbook of Hubei in 2019), the quantity of emergency materials to be distributed to each region of Hubei Province is shown in Table 7:

Table 7. Quantity of materials required in each city

\begin{tabular}{|c|c|c|c|c|c|}
\hline & City & Quantity & & City & Quantity \\
\hline $\mathbf{3}$ & Huangshi & 478 & $\mathbf{1 0}$ & Xian'ning & 492 \\
\hline $\mathbf{4}$ & Shiyan & 659 & $\mathbf{1 1}$ & Suizhou & 429 \\
\hline $\mathbf{5}$ & Yichang & 800 & $\mathbf{1 2}$ & Enshi & 654 \\
\hline $\mathbf{6}$ & Jingmen & 560 & $\mathbf{1 3}$ & Xiantao & 221 \\
\hline $\mathbf{7}$ & Xiaogan & 952 & $\mathbf{1 4}$ & Qianjiang & 187 \\
\hline $\mathbf{8}$ & Jingzhou & 1082 & $\mathbf{1 5}$ & Tianmen & 246 \\
\hline $\mathbf{9}$ & Huanggang & 1225 & $\mathbf{1 6}$ & Shennongjia & 15 \\
\hline
\end{tabular}

Table 6. Distance between the logistics center and each material

demand point and the distance between each material demand point

\begin{tabular}{|l|l|l|l|l|l|l|l|l|l|l|l|l|l|l|l|l|}
\hline & $\mathbf{1}$ & $\mathbf{2}$ & $\mathbf{3}$ & $\mathbf{4}$ & $\mathbf{5}$ & $\mathbf{6}$ & $\mathbf{7}$ & $\mathbf{8}$ & $\mathbf{9}$ & $\mathbf{1 0}$ & $\mathbf{1 1}$ & $\mathbf{1 2}$ & $\mathbf{1 3}$ & $\mathbf{1 4}$ & $\mathbf{1 5}$ & $\mathbf{1 6}$ \\
\hline $\mathbf{1}$ & & & 405.9 & 170.7 & 251.9 & 131.3 & 257.9 & 204.4 & 382.2 & 415 & 167.1 & 450.4 & 326.3 & 265 & 262.5 & 229.9 \\
\hline $\mathbf{2}$ & & & 33.5 & 515.1 & 389.1 & 309.8 & 160.7 & 286.4 & 14 & 122.3 & 244.6 & 588.1 & 167.4 & 221.5 & 210.9 & 560.9 \\
\hline $\mathbf{3}$ & & & & 534.9 & 411.1 & 331.8 & 180.1 & 308.3 & 46.2 & 100.9 & 264.4 & 610.1 & 189.4 & 243.5 & 232.8 & 582.9 \\
\hline $\mathbf{4}$ & & & & & 356.8 & 291.9 & 386.4 & 365 & 510.7 & 525.4 & 295.2 & 555.3 & 456.4 & 425.6 & 407.7 & 194.5 \\
\hline $\mathbf{5}$ & & & & & & 125.3 & 327.4 & 115.4 & 410.7 & 339.5 & 330.3 & 219.7 & 228.6 & 175.8 & 227.9 & 259.5 \\
\hline $\mathbf{6}$ & & & & & & & 227 & 90.9 & 310.3 & 301.5 & 229.4 & 324.7 & 204.6 & 151.4 & 144.8 & 275.9 \\
\hline $\mathbf{7}$ & & & & & & & & 244.9 & 153.5 & 146.5 & 115.5 & 526.9 & 133.3 & 194.9 & 128.2 & 418 \\
\hline $\mathbf{8}$ & & & & & & & & & 307.8 & 238.3 & 263.5 & 314.7 & 127.5 & 74.7 & 126.8 & 307.9 \\
\hline $\mathbf{9}$ & & & & & & & & & & 134 & 251.5 & 614.1 & 193.4 & 247.6 & 237.5 & 554.1 \\
\hline $\mathbf{1 0}$ & & & & & & & & & & & 253.7 & 538.3 & 117.7 & 171.8 & 164 & 531.5 \\
\hline $\mathbf{1 1}$ & & & & & & & & & & & & 529.9 & 191.1 & 197.5 & 150.9 & 327.4 \\
\hline $\mathbf{1 2}$ & & & & & & & & & & & & & 427.9 & 375.2 & 444.8 & 459.1 \\
\hline
\end{tabular}




\begin{tabular}{|l|l|l|l|l|l|l|l|l|l|l|l|l|l|l|l|l|}
\hline $\mathbf{1 3}$ & & & & & & & & & & & & & & 61.5 & 75.3 & 421.2 \\
\hline $\mathbf{1 4}$ & & & & & & & & & & & & & & & 61 & 367.8 \\
\hline $\mathbf{1 5}$ & & & & & & & & & & & & & & & 395.1 \\
\hline
\end{tabular}

\subsection{Results}

According to the designed multi-objective genetic algorithm, python language is used to program the algorithm. The population size is 300 , the evolution algebra is 1000 , the crossover probability is 0.9 , and the mutation probability is 0.1 . The single objective optimization values of each optimization objective are shown in Table 8:

Table 8. The single objective optimization values of each optimization objective

\begin{tabular}{|c|c|}
\hline Objectives of optimization & Quantity \\
\hline Total transport time & 27.383 \\
\hline Total material satisfaction & 13.589 \\
\hline
\end{tabular}

The scheme with the shortest total transportation time is shown in Table 9:

Table 9. Transportation plan with the shortest time

\begin{tabular}{|c|c|c|c|}
\hline $\mathbf{K}$ & $\mathbf{M}$ & Transportation route & Transportation time \\
\hline $\mathbf{1}$ & 1 & $1-5-12$ & 5.895 \\
\hline $\mathbf{2}$ & 2 & $2-9$ & 0.175 \\
\hline $\mathbf{3}$ & 2 & $2-3-9$ & 0.996 \\
\hline $\mathbf{4}$ & 2 & $2-15-8$ & 4.221 \\
\hline $\mathbf{5}$ & 1 & $1-6-8$ & 2.778 \\
\hline $\mathbf{6}$ & 1 & $1-11$ & 2.089 \\
\hline $\mathbf{7}$ & 2 & $2-13-14$ & 2.861 \\
\hline $\mathbf{8}$ & 1 & $1-4$ & 2.133 \\
\hline $\mathbf{9}$ & 2 & $2-10-7$ & 3.360 \\
\hline $\mathbf{1 0}$ & 1 & $1-16$ & 2.874 \\
\hline
\end{tabular}

Through repeated debugging of relevant parameters, a relatively better solution of material satisfaction at each material demand point is obtained, as shown in Table 10 (Obj2 indicates the actual material satisfaction):

Table 10. Material satisfaction of each material demand point

\begin{tabular}{|c|c|c|c|}
\hline City & Obj2 & City & Obj2 \\
\hline Huangshi & $100 \%$ & Xian'ning City & $100 \%$ \\
\hline Shiyan & $100 \%$ & Suizhou & $100 \%$ \\
\hline Yichang & $100 \%$ & Enshi & $100 \%$ \\
\hline Jingmen & $100 \%$ & Xiantao & $100 \%$ \\
\hline Xiaogan & $100 \%$ & Qianjiang & $100 \%$ \\
\hline Jingzhou & $100 \%$ & Tianmen & $100 \%$ \\
\hline Huanggang & $41.06 \%$ & Shennongjia & $100 \%$ \\
\hline
\end{tabular}

\section{Conclusions}

In this paper, an emergency logistics LRP model based on public health emergencies is proposed. Then Taking transportation time and material satisfaction as the optimization goal, NSGA-II algorithm is introduced to solve the model, and python language is used to program the algorithm.
In the future research, it is proposed to take into account the number of infected people, the number of deaths, the situation of material shortage and other factors, and to use fuzzy cluster analysis and other methods for more reasonable distribution of materials.

\section{References}

1. M. TAVANA, A. ABTAHI, D. Di CAPRIO, et al. An integrated location-inventory-routing humanitari an supply chain network with pre- and post-disaster management considerations[J], Socio-Economic Planning Sciences, 64: 21-37 (2018).

2. W. Li, Research on LRP problem of emergency logistics system considering priority [D], Beijing : Beijing Jiaotong University (2019) .

3. H. Wang, L. Du, S. Ma, Multi-objective open location-routing model with split delivery for optimized relief distribution in post-earthquake[J]. Transportation Research, 69 : 160-179 (2014).

4. G. Chen, Study on the LRP of Post-Earthquake Emergency Logistics Considering Distribution Priority and Highway State [D]. Harbin: Northeast Agricultural University (2016).

5. Y. Zhou, Y. Zhang, et al. A multi-objective evolutionary algorithm for multi-period dynamic emergency resource scheduling problems[J]. Transportation Research Part E: Logistics and Transportation Review, 99: 77-95 (2017).

6. J. Xu, Z. Wang, M. Zhang, et al. A new model for a 72-h post-earthquake emergency logistics locationrouting problem under a random fuzzy environment[J]. Transportation Letters : The International Journal of Transportation Resear,8: 270-285 (2016).

7. B. VAHDANI, D. VEYSMORADI, N. SHEKARI, et al. Multi-objective, multi-period location-routing model to distribute relief after earthquake by considering emergency roadway repair[J]. Neural Computing and Applications, 30(3): 835-854 (2018).

8. Q. Li, W. Tu,L. Zhuo. Reliable Rescue Routing Optimization for Urban Emergency Logistics under Travel Time Uncertainty[J]. ISPRS International Journal of Geo-Information,,7(2): 77 (2018).

9. L. Shen, F. Tao, Y. Shi, et al. Optimization of Location-Routing Problem in Emergency Logistics Considering Carbon Emissions[J]. Int J Environ Res Public Health,16 (2019).

10. A. Moreno, D. Alem, D. Ferreira. Heuristic approaches for the multiperiod locationtransportation problem with reuse of vehicles in emergency logistics $[\mathrm{J}]$. Computers \& Operations Research,69: 79-96 (2016).

11. K. Deb, A. Pratap, S. Agrawal, et al. A Fast and Elitist Multi-Objective Genetic Algorithm: NSGAII [R]. KanGAL Report No. 200001 (2000). 